\title{
DWI Lesion Patterns in Cancer-Related Stroke - Specifying the Phenotype
}

\author{
Christopher Jan Schwarzbach Marc Fatar Philipp Eisele Anne D. Ebert \\ Michael G. Hennerici Kristina Szabo \\ Department of Neurology, University Medical Centre Mannheim, Heidelberg University, \\ Mannheim, Germany
}

\section{Key Words}

Stroke and cancer - Acute stroke - Recurrent stroke - Embolic stroke - Cerebral embolism . Diagnostic criteria $\cdot$ MRI-DWI $\cdot$ Risk factors for stroke

\begin{abstract}
Background: Due to the lack of specific diagnostic markers, the diagnosis of cancer-related stroke strongly depends on its phenotype. Distinct DWI lesion patterns with involvement of multiple vascular territories have been reported repeatedly in cancer-related stroke but have not been addressed in detail in a selected cohort of prospectively recruited cancer patients with emphasis on hypercoagulable conditions. Patients and Methods: Ischemic stroke patients with known malignant cancer activity, laboratory evidence of strong plasmatic hypercoagulation (D-dimer levels $>3 \mu \mathrm{g} / \mathrm{ml}$ ) and without competing stroke etiologies according to the recently introduced ASCOD ( $\mathrm{A}$ - atherosclerosis, $\mathrm{S}$ - small vessel disease, $\mathrm{C}$ - cardiac pathology, $\mathrm{O}$ - other cause, and D - dissection) classification of evidence-rated etiology of stroke subtypes were included in the analysis. Cerebral MRI on admission was reviewed with respect to ischemic lesion patterns. Results: Thirty-two patients met the inclusion criteria. The mean D-dimer levels were $15.39 \mu \mathrm{g} / \mathrm{ml}( \pm 10.84)$. Acute infarction in $\geq 2$ vascular territories was present in 27/32 (84\%) patients. (Micro-) embolic scattering of infarction was present in 25/32 (78\%) patients. Evidence for previous, potentially oligosymptomatic infarction was found in $16(50 \%)$ patients, demonstrated by the additional presence of subacute or chronic ischemic lesions. Conclusion: When excluding competing embolic and nonembolic stroke etiologies, the pattern of scattered DWI lesions in multiple vascular supply territories strongly dominates
\end{abstract}

The abstract and presentation of this paper was awarded by the European Stroke Research Foundation during the 24th European Stroke Conference 2015 in Vienna for outstanding contribution to current stroke research. 
the phenotype of cancer-related stroke. Additionally, evidence of recurrent infarction is frequent in this cohort of patients. This is not only important for the diagnosis of cancer-related stroke itself but may prove helpful for the identification of cancer-related stroke patients with unknown malignancy at the time of stroke manifestation and evaluation of strategies for secondary prevention.

\section{Background}

Ischemic stroke and cancer represent a frequent coincidence with large-scale significance on stroke etiology, diagnostics and treatment options. Evidence from early autopsy and recent clinical studies suggest a prevalence of ischemic stroke in cancer patients of approximately 3\% [1, 2]. Considering the annual incidence of cancer in Germany published by the Robert Koch Institute in 2012, this number would imply 15,000 affected stroke patients in Germany each year [3]. In practice, however, these numbers are much smaller as the association of stroke and cancer often remains unappreciated. This is due to the fact that cancerrelated stroke is underestimated and difficult to diagnose, in particular in patients without a known cancer history or unrecognized stroke subtype patterns. Owing to the lack of specific diagnostic markers, the diagnosis of cancer-related stroke strongly depends on its phenotype, which is determined by the interrelation of cancer-related hypercoagulation and concomitant arterial embolism [4, 5].

DWI-MRI is exquisitely sensitive to detect areas of acute ischemic tissue change [6] and has improved the understanding of distinct pathophysiological mechanisms leading to ischemia in several subgroups of stroke with differences in patterns considering lesion size, localization and distribution [7-9]. Specifying DWI lesions in cancer-related stroke may therefore amplify our understanding of the pathophysiological mechanisms, which take effect in patients with cancer-related stroke and, moreover, may be easily applied to help identifying patients affected by cancer-related stroke mechanisms.

On the basis of the contemporary pathophysiological perception of cancer-related stroke, we hypothesized that embolic stroke patterns would be dominant in this selected cohort of stroke patients.

\section{Patients and Methods}

\section{Patient Selection}

Patients with acute ischemic stroke, the additional diagnosis of solid and active malignancy, laboratory evidence of strong plasmatic hypercoagulation (D-dimer levels $>3 \mu \mathrm{g} / \mathrm{ml}$ ) and without competing stroke etiologies according to the ASCOD (A - atherosclerosis, $\mathrm{S}$ - small vessel disease, $\mathrm{C}$ - cardiac pathology, $\mathrm{O}$ - other cause, and D - dissection) classification of evidence-rated etiology of stroke subtypes [10] were included in the analysis. Participants were recruited from our comprehensive stroke center (Department of Neurology, University Medical Centre Mannheim, Heidelberg University, Mannheim, Germany) and identified by our prospectively collected stroke data bank for the years 2004-2014. Active cancer was defined as confirmed malignancy treated or untreated in the last 6 months before stroke. Diagnosis of cancer was confirmed by given medical records or, in case of newly diagnosed or recurrent cancer, by histological evidence and oncologist expertise. Patients with hematologic malignancies or primary brain tumor were not included in the study because these patients were considered to represent a subgroup with different underlying stroke mechanisms. 


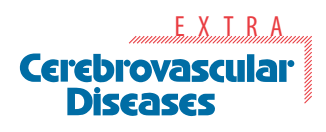

Cerebrovascul
Diseases

\begin{tabular}{l|l}
\hline Cerebrovasc Dis Extra 2015;5:139-145 \\
\hline DOI: 10.1159/000439549 & $\begin{array}{l}\text { C } 2015 \text { The Author(s). Published by S. Karger AG, Basel } \\
\text { www.karger.com/cee }\end{array}$ \\
\hline
\end{tabular}

Schwarzbach et al.: DWI Lesion Patterns in Cancer-Related Stroke - Specifying the Phenotype

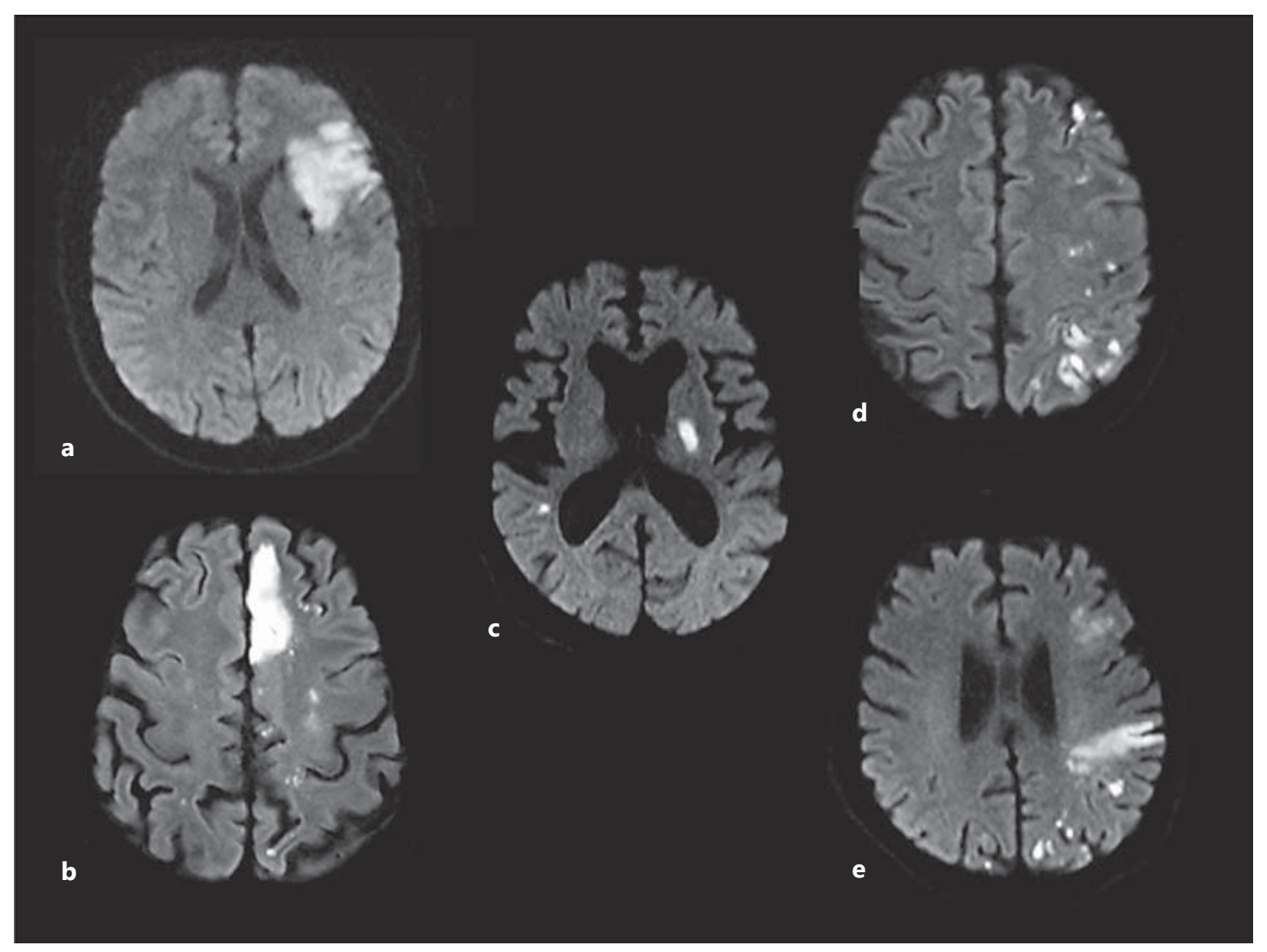

Fig. 1. Characterization of DWI lesion patterns according to number and localization. a Single acute lesion. b Multiple acute lesions in one vascular territory with (micro-) embolic scattering of infarction. c Multiple acute lesions in $>1$ vascular territory (bihemispheric anterior circulation lesions) without (micro-) embolic scattering of infarction. $\mathbf{d}$ Multiple acute lesions in $>1$ vascular territory (bihemispheric anterior circulation lesions) with (micro-) embolic scattering of infarction. e Multiple acute lesions in $>1$ vascular territory (anterior and posterior circulation lesions) with (micro-) embolic scattering of infarction.

\section{Clinical Management and Data Acquisition}

Type of cancer, stroke etiology and D-dimer levels were identified consistently by reviewing each patient. D-dimer levels were assessed at the time of hospitalization. Full stroke workup included extra- and intracranial Doppler and duplex sonography of brainsupplying arteries, electrocardiography on admission, $72 \mathrm{~h}$ of electrocardiographic and vital sign monitoring, transthoracic or transesophageal echocardiography and laboratory tests (routine hematology and biochemistry, including coagulation test) as standard procedures according to stroke unit management recommendations (European Stroke Organization Guidelines, 2008). Patients without sufficient MRI at the time of hospitalization were excluded from the analysis.

\section{MRI Assessment and Analysis}

The MRI investigations were performed on 1.5-tesla and 3.0-tesla MRI scanners (MAGNETOM Sonata, Trio or Skyra; Siemens, Erlangen, Germany). The scanning protocol included transversal DWI, T2-weighted fluid-attenuated inversion recovery, T1- and T2-weighted images, obtained with a 5-mm slice thickness, and an MR angiography. The acute lesion pattern on DWI was characterized according to number and localization with regard to the affected vascular territory (fig. 1). The presence of (micro-) embolic lesions was 
documented, defined as $>5$ scattered small DWI lesions (fig. 1). In addition, subacute (DWI hyper- and apparent diffusion coefficient iso- or hyperintense) and chronic ischemic lesions were assessed. Images were reviewed independently by two stroke neurologists (C.J.S. and K.S.). In cases of discrepancy, the final pattern classification was reached by mutual agreement of the readers.

\section{Stroke Etiology}

Before inclusion, the patients were phenotypically characterized according to the ASCOD classification [10]. The prior ASCO phenotypic classification of stroke has shown good concordance with the most widely used Trial of Org 10172 in Acute Stroke Treatment classification [11]. Additionally, the ASCOD classification reflects important supplementary information. Patients with a competing stroke mechanism, defined by the presence of an ASCOD phenotype A (for atherothrombosis), S (for small vessel disease), C (for cardiac pathology), 0 (for other causes) or D (for dissection) graded 1 or 2 were excluded from the analysis. With respect to the suspected cancer-related stroke mechanisms, exceptions were made for patients with 'no direct cardiac source identified, but multiple brain infarction, repeated either bilateral or in two different arterial territories (e.g. both anterior and posterior circulation)', classified C2 according to the ASCOD classification, and nonbacterial thrombotic 'endocarditis', classified C1 according to the ASCOD classification.

\section{Ethical Considerations}

The study was approved by the local institutional ethics committee (Medizinische Ethikkomission II der Medizinischen Fakultät Mannheim der Ruprecht-Karls-Universität Heidelberg).

\section{Results}

\section{Patient Characteristics and D-Dimer Assessment}

Thirty-two patients (16 male, 16 female) met the inclusion criteria and were included in the analysis. The mean age was 68.7 years. Lung carcinoma was most frequently observed $(\mathrm{n}=13,40 \%)$, followed by colorectal carcinoma $(\mathrm{n}=4,12.5 \%)$, gastric carcinoma $(n=4$, $12.5 \%$ ) and cancer of an unknown primary (CUP; $n=4,12.5 \%)$. Three patients (9\%) suffered from pancreatic carcinoma, 2 (6\%) from prostate cancer and 1 patient each from renal $(3 \%)$ and ovarian $(3 \%)$ carcinoma. None of the included patients or control subjects showed imaging evidence or clinical syndrome suggestive of cerebral vein thrombosis or primary intracerebral hemorrhage as these patients were not considered in the study per se. Metastatic disease, earlier considered as an additional stroke risk factor [4], was present in 29/32 $(90 \%)$ patients.

The mean D-dimer levels were assessed with a routine coagulation test and were 15.39 $\mu \mathrm{g} / \mathrm{ml}( \pm 10.84)$ after a lower cutoff value of $3 \mu \mathrm{g} / \mathrm{ml}$ had been defined for patient inclusion. The time between admittance to hospital and D-dimer assessment was 2.1 days $( \pm 2.2)$ on average.

\section{MRI Categorization}

MRI demonstrated acute ischemic lesions in $\geq 2$ vascular territories in $27 / 32$ (84\%) patients. Additionally, (micro-) embolic scattering of infarction, as visualized in figure 1 , was present in 25/32 (78\%) patients. Examples of DWI lesion patterns, characterized according to number and localization, are given in figure 1 . The results of acute ischemic lesion patterns are summarized in detail in table 1. 


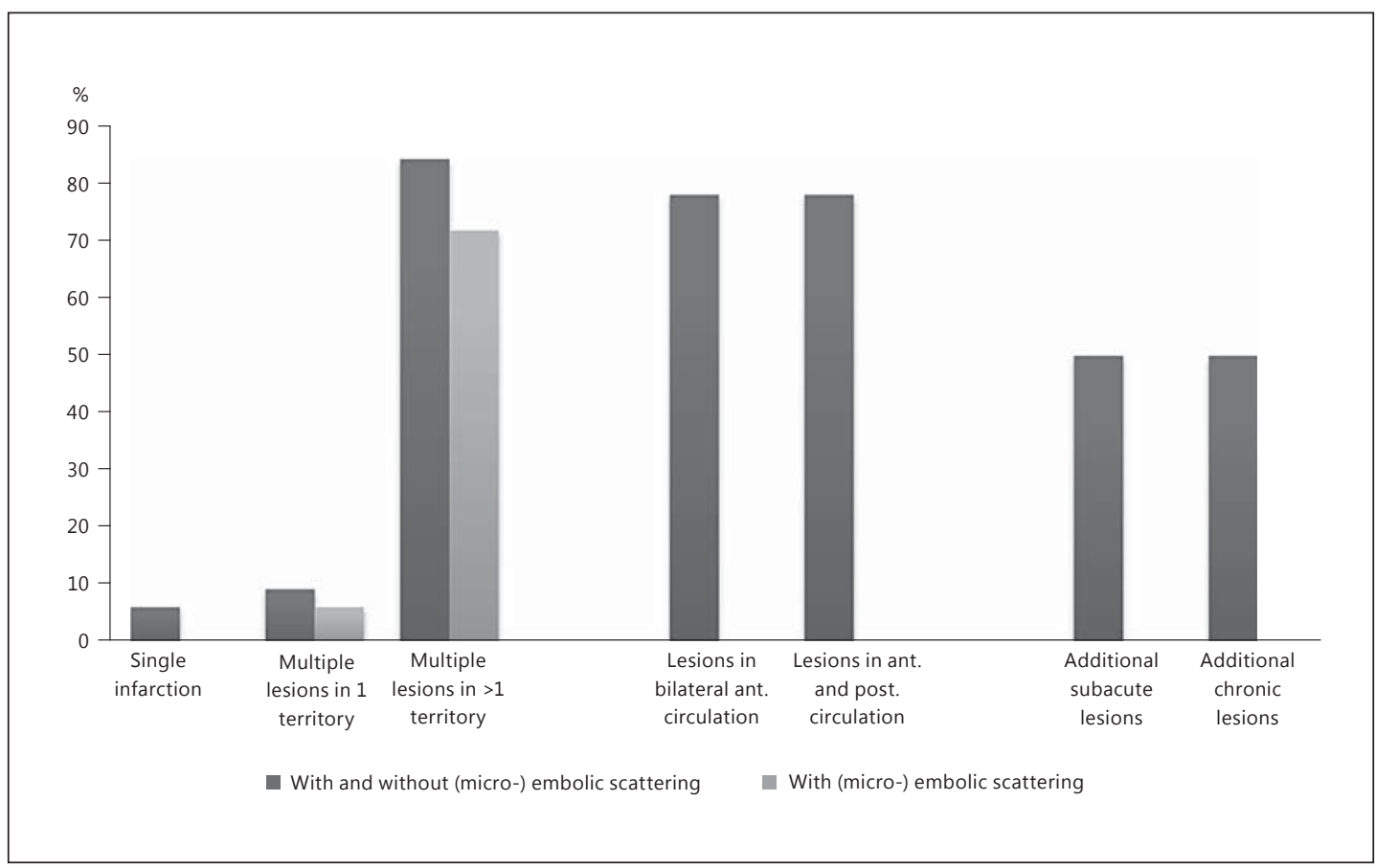

Fig. 2. Distribution of ischemic lesion patterns. Categorized into 'Single infarction', 'Multiple lesions in one vascular territory' and 'Multiple lesions in >1 vascular territory'. The frequency of lesions on 'bilateral anterior circulation' and 'anterior and posterior circulation' is additionally visualized as well as the frequency of 'additional subacute lesions' and 'chronic lesions'.

Table 1. MRI categorization

\begin{tabular}{llllr}
\hline & $\begin{array}{l}\text { Single } \\
\text { infarction, } \\
\mathrm{n}\end{array}$ & $\begin{array}{l}\text { Multiple acute } \\
\text { lesions in 1 } \\
\text { territory, } \\
\mathrm{n}\end{array}$ & $\begin{array}{l}\text { Multiple acute } \\
\text { lesions in }>1 \\
\text { vascular territory, } \\
\mathrm{n}\end{array}$ & $\begin{array}{l}\text { Total, } \\
\mathrm{n}\end{array}$ \\
\hline Total & 2 & 3 & 27 & 32 \\
Lesions in anterior and posterior circulation & 0 & 0 & 25 & 25 \\
Lesions in bilateral anterior circulation & 0 & 0 & 25 & 25 \\
Additional subacute lesions in the same territory & 0 & 0 & 12 & 12 \\
Additional subacute lesions in an additional territory & 1 & 0 & 3 & 4 \\
Additional chronic lesions in the same territory & 0 & 0 & 11 & 5 \\
Additional chronic lesions in an additional territory & 0 & 2 & 3 & 3 \\
Cerebral metastasis & 0 & 0 & 23 & 25 \\
Additional (micro-) embolic scattering & 0 & 2 & 25 \\
\hline
\end{tabular}

As an indication of recurrent strokes, 16/32 (46\%) patients presented with additional subacute infarction. The same number of patients $(16 / 32,46 \%)$ also showed evidence of chronic infarction. The distribution of lesion patterns is additionally visualized in figure 2 .

Three patients $(8.5 \%)$ showed additional evidence of cerebral metastasis. In all of these cases, ischemic lesions could be easily distinguished from cerebral metastasis. One patient showed extensive cerebral metastasis and was therefore excluded from the analysis. 


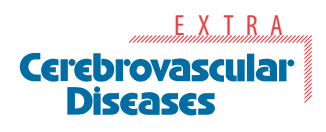

\begin{tabular}{l|l}
\hline Cerebrovasc Dis Extra 2015;5:139-145 \\
\hline DOI: $10.1159 / 000439549$ & $\begin{array}{l}\text { C } 2015 \text { The Author(s). Published by S. Karger AG, Basel } \\
\text { www.karger.com/cee }\end{array}$ \\
\hline
\end{tabular}

Schwarzbach et al.: DWI Lesion Patterns in Cancer-Related Stroke - Specifying the Phenotype

\section{Discussion}

As the diagnosis of cancer-related stroke is strongly dependent on its phenotype, its specification is of highest importance. Especially the lack of recognition of a possibly cancerrelated ischemic stroke pattern may prevent the identification of the present cancer-related stroke mechanisms and may be a reason for the undervaluation of this important phenomenon in cancer patients with stroke. Currently, evidence of hypercoagulation and the presence of active malignancy in the absence of conventional stroke mechanisms such as atrial fibrillation or large vessel disease dominate the diagnostic criteria of cancer-related ischemic stroke [10]. Looking at these diagnostic criteria, DWI lesion patterns have received little attention, even though distinct DWI lesion patterns with involvement of multiple vascular territories have been reported repeatedly in cancer-related stroke $[4,12,13]$. This may be due to the fact that these lesion patterns have never been addressed in detail in a selected cohort of cancer patients with evidence of hypercoagulation before. Our data shows that after the exclusion of competing embolic and nonembolic stroke etiologies, DWI lesions in multiple vascular supply territories strongly dominate the phenotype of cancer-related stroke, being observed in $84 \%$ of all patients included in this analysis. Additionally, (micro-) embolic scattering is a frequent feature of ischemic infarction in this special cohort of patients (being observed in $78 \%$ of all patients included in this analysis). This is well in line with previous results showing an elevated prevalence of embolic signal detected by transcranial Doppler monitoring in cancer patients in up to 58\% and associated with increased D-dimer levels [14]. Our results also confirm the common pathophysiological perception of recurrent cerebral (micro-) embolization due to cancer-related hypercoagulation as leading cause of cancer-related ischemic stroke.

Furthermore, our data shows that signs of recurrent embolization on MRI by the detection of infarction at variable stages (acute, subacute or chronic) are also common in cancer patients with evidence of hypercoagulation and without competing stroke etiologies. This is consistent with other data, reporting an increased risk of new DWI lesions in patients with multiple territory lesions at initial presentation [15]. As multiple DWI lesions have been shown to be associated with an increased risk of recurrent stroke [16], this finding may emphasize the urgency of validated strategies for secondary prevention of ischemic stroke in these patients. This conclusion supports preliminary findings concerning the elevated risk of recurrent stroke in cancer patients $[17,18]$. However, these strategies still need to be established by prospective, randomized and controlled data. In the meantime, in selected individuals, the strategy for secondary prevention may be deduced from data concerning secondary prevention of venous thromboembolism in cancer patients $[19,20]$. With respect to this data, treatment with low-molecular-weight heparin is currently recommended as first-line therapy by the engaged medical societies [21].

In conclusion, (micro-) embolic scattering of DWI lesions in multiple vascular supply territories and in variable stages appear to be an important aspect of the phenotype of cancerrelated stroke and are worth being considered in the diagnostic criteria. These results are not only important for the diagnosis of cancer-related stroke itself but potentially also for identifying cancer-related stroke patients with unknown malignancy at the time of stroke manifestation, which, however, remains to be addressed by a separate prospective approach.

\section{Disclosure Statement}

None of the authors have a conflict of interest related to the present study. 


\section{Cerebrovascular’ Diseases}

\begin{tabular}{l|l}
\hline Cerebrovasc Dis Extra 2015;5:139-145 \\
\hline DOI: 10.1159/000439549 & $\begin{array}{l}\text { C } 2015 \text { The Author(s). Published by S. Karger AG, Basel } \\
\text { www.karger.com/cee }\end{array}$ \\
\hline
\end{tabular}

Schwarzbach et al.: DWI Lesion Patterns in Cancer-Related Stroke - Specifying the Phenotype

\section{References}

-1 Graus F, Rogers LR, Posner JB: Cerebrovascular complications in patients with cancer. Medicine (Baltimore) $1985 ; 64: 16-35$

2 Chen PC, Muo CH, Lee YT, Yu YH, Sung FC: Lung cancer and incidence of stroke: a population-based cohort study. Stroke 2011;42:3034-3039.

3 Krebs in Deutschland 2007/2008, ed. 8. Berlin, Robert Koch-Institut und die Gesellschaft der epidemiologischen Krebsregister in Deutschland e.V., 2012.

-4 Schwarzbach CJ, Schaefer A, Ebert A, Held V, Bolognese M, Kablau M, Hennerici MG, Fatar M: Stroke and cancer: the importance of cancer-associated hypercoagulation as a possible stroke etiology. Stroke 2012;43:3029 3034.

5 Bang OY, Seok JM, Kim SG, Hong JM, Kim HY, Lee J, Chung PW, Park KY, Kim GY, Chung CS, Lee KH: Ischemic stroke and cancer: stroke severely impacts cancer patients, while cancer increases the number of strokes. J Clin Neurol 2011;7:53-59.

6 Albers GW, Lansberg MG, Norbash AM, Tong DC, O’Brien MW, Woolfenden AR, Marks MP, Moseley ME: Yield of diffusion-weighted MRI for detection of potentially relevant findings in stroke patients. Neurology 2000; 54:1562-1567.

7 Szabo K, Kern R, Gass A, Hirsch J, Hennerici M: Acute stroke patterns in patients with internal carotid artery disease: a diffusion-weighted magnetic resonance imaging study. Stroke 2001;32:1323-1329.

8 Rovira A, Grivé E, Rovira A, Alvarez-Sabin J: Distribution territories and causative mechanisms of ischemic stroke. Eur Radiol 2005;15:416-426.

-9 Gass A, Ay H, Szabo K, Koroshetz WJ: Diffusion-weighted MRI for the 'small stuff': the details of acute cerebral ischaemia. Lancet Neurol 2004;3:39-45.

10 Amarenco P, Bogousslavsky J, Caplan LR, Donnan GA, Wolf ME, Hennerici MG: The ASCOD phenotyping of ischemic stroke (Updated ASCO Phenotyping). Cerebrovasc Dis 2013;36:1-5.

11 Wolf ME, Sauer T, Alonso A, Hennerici MG: Comparison of the new ASCO classification with the TOAST classification in a population with acute ischemic stroke. J Neurol 2012;259:1284-1289.

12 Kim SG, Hong JM, Kim HY, Lee J, Chung PW, Park KY, Kim GM, Lee KH, Chung CS, Bang OY: Ischemic stroke in cancer patients with and without conventional mechanisms: a multicenter study in Korea. Stroke 2010;41: 798-801.

13 Lee EJ, Nah HW, Kwon JY, Kang DW, Kwon SU, Kim JS: Ischemic stroke in patients with cancer: is it different from usual strokes? Int J Stroke 2014;9:406-412.

14 Seok JM, Kim SG, Kim JW, Chung CS, Kim GM, Lee KH, Bang OY: Coagulopathy and embolic signal in cancer patients with ischemic stroke. Ann Neurol 2010;68:213-219.

15 Braemswig TB, Usnich T, Albach FN, Brunecker P, Grittner U, Scheitz JF, Fiebach JB, Nolte CH: Early new diffusion-weighted imaging lesions appear more often in stroke patients with a multiple territory lesion pattern. Stroke 2013;44:2200-2204.

-16 Kang DW, Lattimore SU, Latour LL, Warach S: Silent ischemic lesion recurrence on magnetic resonance imaging predicts subsequent clinical vascular events. Arch Neurol 2006;63:1730-1733.

-17 Lau KK, Wong YK, Teo KC, Chang RS, Hon SF, Chan KH, Cheung RT, Li LS, Tse HF, Ho SL, Siu CW: Stroke patients with a past history of cancer are at increased risk of recurrent stroke and cardiovascular mortality. PLoS One 2014;9:e88283.

18 Kim JM, Jung KH, Park KH, Lee ST, Chu K, Roh JK: Clinical manifestation of cancer related stroke: retrospective case-control study. J Neurooncol 2013;111:295-301.

-19 Akl EA, Labedi N, Barba M, Terrenato I, Sperati F, Muti P, Schünemann H: Anticoagulation for the long-term treatment of venous thromboembolism in patients with cancer. Cochrane Database Syst Rev 2011:CD006650.

20 Akl EA, Vasireddi SR, Gunukula S, Barba M Sperati F, Terrenato I, Muti P, Schünemann H: Anticoagulation for the initial treatment of venous thromboembolism in patients with cancer. Cochrane Database Syst Rev 2011:CD006649.

-21 Lyman GH, Bohlke K, Khorana AA, Kuderer NM, Lee AY et al: Venous thromboembolism prophylaxis and treatment in patients with cancer: American Society of Clinical Oncology clinical practice guideline update 2014. J Clin Oncol 2015;33:654-656. 